\title{
Flexible Multi-Electrode Array for Medical Applications
}

\author{
Nadine Winkin, Wilfried Mokwa \\ Institute of Materials in Electrical Engineering 1, RWTH Aachen University, Sommerfeldstr. 24, 52074 Aachen, Germany
}

Corresponding author: winkin@iwe1.rwth-aachen.de

\begin{abstract}
A flexible multi-electrode array (MEA) with an embedded silicon chip for electrical stimulation of neurons or for recording action potentials has been manufactured and characterized. Possible improvements for medical applications using this novel approach are presented. By connecting and addressing several of these MEAs via a bus system, the number and the density of electrodes can be increased significantly. This is interesting for medical applications such as retinal implants and cochlear implants, and also for deep brain stimulators. Design and fabrication techniques for the multi-electrode array are presented. Finally, first results of mechanical stress tests are shown.
\end{abstract}

Keywords: flexible multi-electrode array, thinned silicon chip, electrical stimulation, retinal implant.

\section{Introduction}

Micro-electrodes for stimulating neurons or for recording action potentials are widely used in medical applications. This paper concentrates on the example of an intraocular implant.

Worldwide more than 3 million people suffer from Retinitis Pigmentosa (RP). This hereditary disease leads to gradual degeneration of the light-sensitive cells (rods and cones) of the retina. As a result, the field of sight becomes smaller over the years, and in most cases, this disease ends up in total blindness. Unfortunately, RP is still incurable. However, it has been shown, that even after many years of blindness, about $30 \%$ of the retinal ganglion cells of RP patients are still intact. Electrical stimulation of these remaining cells can evoke visual perceptions $[1,2,3,9]$. Several research groups worldwide are working on the development of epiretinal implants for stimulating these remaining intact ganglion cells.

One electrical stimulation concept that has been realized is the "EPI-RET-3" wireless-powered implant system funded by the German Ministry of Education and Research (BMBF). This system consists of an extraocular part and an intraocular part, see Fig. 1. A portable computer system, a transmitter unit and a transmitter coil form the extraocular part. The transmitter coil is attached to a special holder similar to an eyeglass frame.

The intraocular part — the epiretinal implant consists of a flexible micro cable with an integrated receiver coil, a receiver chip, a stimulation chip, and stimulation electrodes. The electrode array with 25 electrodes is placed directly onto the retina and is fixed with retinal tacks. The electrode array is connected via conductor tracks with the electronic components located in an artificial lens in the posterior chamber

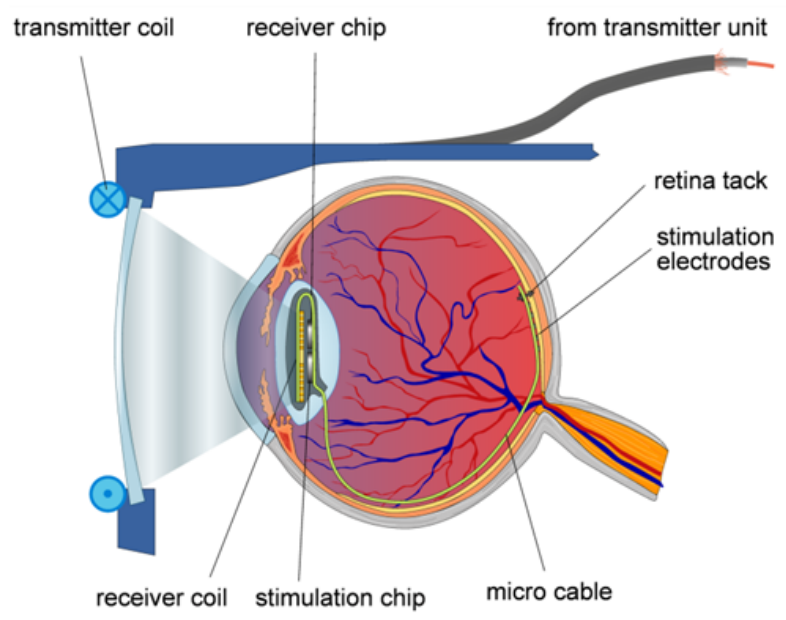

Figure 1: Schematic view of the EPI-RET-3 implant system [3]

of the eye.

This epiretinal implant not only has been tested in animal experiments, but has already been implanted in the eyes of six blind RP patients. They wore the implant for a trial period of four weeks, without any complications. The surgeries for implantation and explantation, and also the stimulation tests, were performed at the eye clinics of the Universities of Aachen and Essen, Germany. The RP patients reported dots, lines, arcs or circles in different colours and intensities, depending on the stimulations $[3,2]$.

The next step will now be to design and develop a second-generation wireless implant system with a considerably higher number of electrodes, increasing the spatial resolution as well as the sight of view [2]. However, it would be a major technical task to contact a microelectrode array with for example 1000 electrodes by separate tracks and to control them 


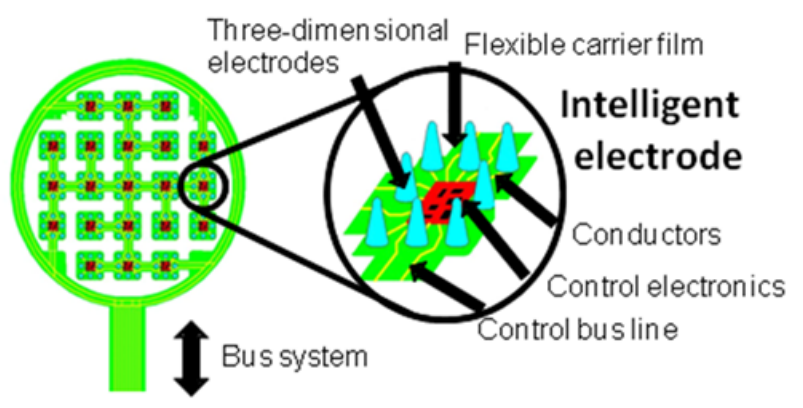

Figure 2: Flexible microelectrode array with distributed control electronics and distributed electrodes.

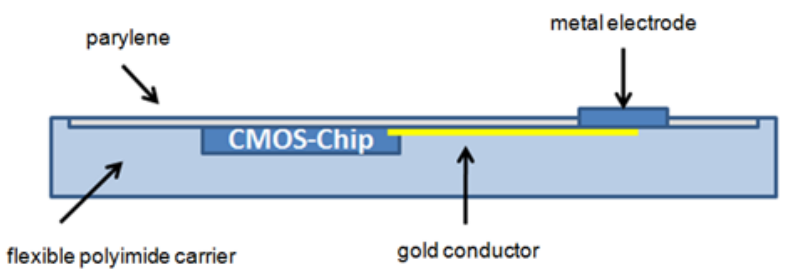

Figure 3: Design of Flex-MEA (schematic).

individually. A feasible approach is to control the electrodes locally with intelligent CMOS circuits, each driving for example 25 electrodes. Several of these circuits could be connected and addressed via a bus system. The whole has to be embedded into a flexible carrier for better adaption to the human eye. The idea is shown in Fig. 2. The following sections will present the design and the fabrication of a "flexible intelligent electrode".

\section{Design and Fabrication}

\subsection{Design}

To obtain the desired flexibility of the CMOS-chips, they have to be thinned to thicknesses below $20 \mu \mathrm{m}$. These thinned CMOS-chips, the gold conductors to the electrodes and 25 electrodes are integrated into a polyimide carrier, and are encapsulated with parylene. In the following text, this structure is called "FlexMEA" (Fig. 3). Flex-MEA will act as the basic unit for the "flexible intelligent electrode".

During the development phase, a dummy stimulator chip was used to optimize integration into the polyimide. This silicon chip has aluminum pads on top, as shown in Fig. 4. The pad dimensions and the spacings are identical to those of the CMOS-chips, used in the EPI-RET-3 implant. The 5 pads on the left side are used for controlling the CMOS-chip, and the 25 pads on the right side are intended for driving the stimulation electrodes.

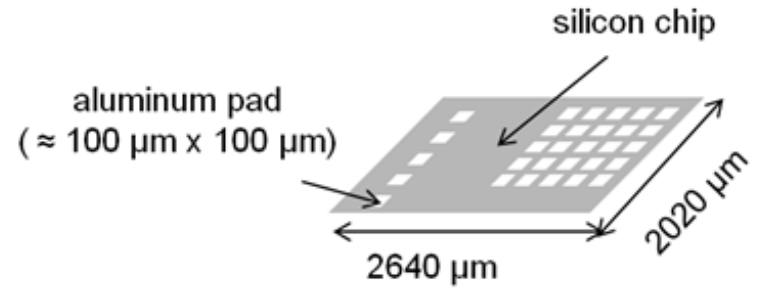

Figure 4: Dummy stimulator chip (schematic).

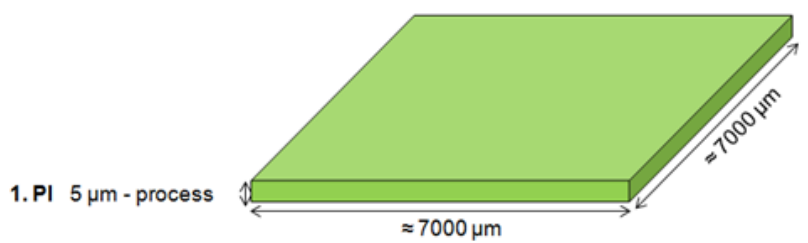

Figure 5: Polyimide base structure of Flex-MEA.

\section{$2.2 \quad$ Fabrication}

Flex-MEA was fabricated in the Institute's own clean room at RWTH Aachen.

The first step was to prepare the polyimide base structure. "PI 2611" liquid polyimide from HD MicroSystems was applied to an alu-sub-wafer using a spin coater. The alu-sub-wafer consists of a silicon wafer coated with $50 \mathrm{~nm}$ titanium, $1 \mu \mathrm{m}$ aluminum and $150 \mathrm{~nm}$ titanium. The first titanium coating serves as an adhesion layer for the aluminum. The aluminum itself serves as a sacrificial layer during the separation of the final prepared polyimide carrier from the wafer. The second titanium coating protects the aluminum from unwanted etching attacks during the processing steps.

PI 2611 has excellent biocompatibility and biostability [7]. Depending on the number of revolutions (rpm) of the spin coater, various layer thicknesses between $1 \mu \mathrm{m}$ and $11 \mu \mathrm{m}$ can be produced. A layer thickness of $5 \mu \mathrm{m}$ was used for the base structure of Flex-MEA. After photolithographic and wet chemical patterning, structures with dimensions of $7000 \times 7000 \times 5 \mu \mathrm{m}$ were created (Fig. 5). Next, the polyimide base structure (1. PI) had to be cured entirely. This is done in an oven at temperatures around $350^{\circ} \mathrm{C}$ to $400^{\circ} \mathrm{C}$ under a nitrogen atmosphere. After this treatment, the PI is resistant to most solvents, acids and bases.

As has already been described, just polyimide layers between $1 \mu \mathrm{m}$ and $11 \mu \mathrm{m}$ in thickness can be prepared. Greater thicknesses of the polyimide layer can be formed by alternating coating and bake-out steps. Based on this approach, IWE1 developed a $25 \mu \mathrm{m}$-process, which was applied here.

In the following step, the $20 \mu \mathrm{m}$ thinned dummy stimulator chip had to be embedded into the polyimide. After photolithographic and wet chemical patterning, a trench of about $2790 \times 2170 \mu \mathrm{m}$ was created 


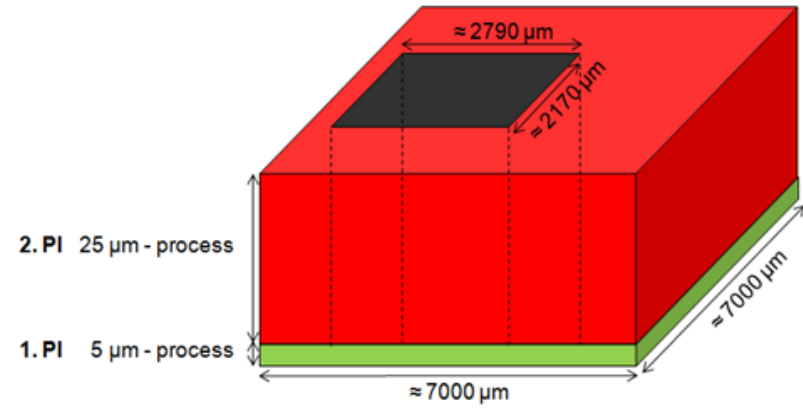

Figure 6: Generating a polyimide trench.

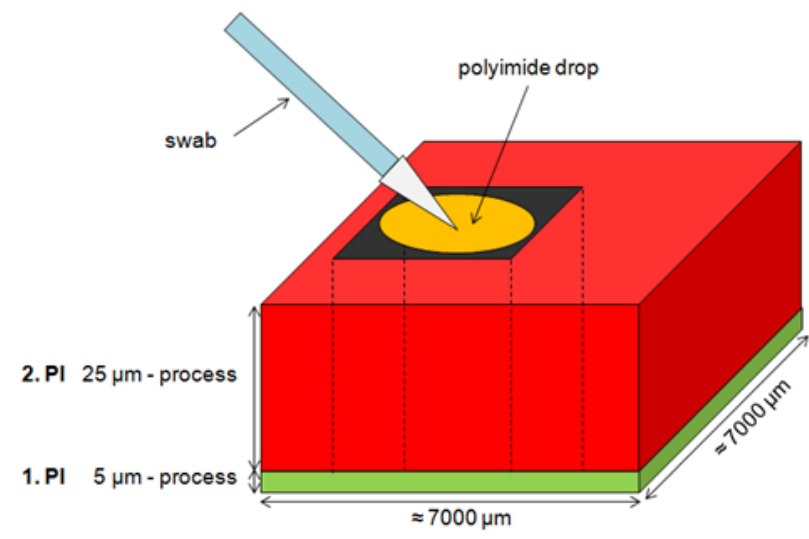

Figure 7: Setting a polyimide drop for fixing the chip.

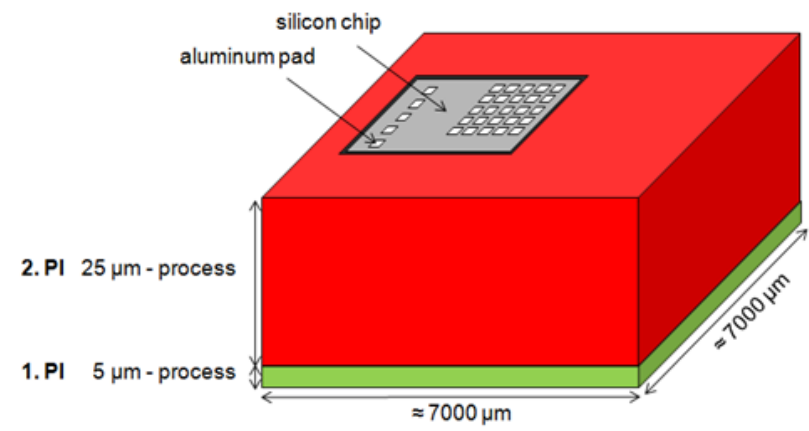

Figure 8: Gluing the dummy stimulator chip into the polyimide.

on the thick polyimide layer (see Fig. 6). To glue and fix the chip, a drop of liquid polyimide was given into the trench using a swab (Fig. 7). In the subsequent step, the chip was placed into the trench, centered and fixed, using tweezers. The glued chip is shown in Fig. 8.

The dummy stimulator chip protruded slightly from the trench. To integrate the chip entirely, an additional layer of $7 \mu \mathrm{m}$ polyimide was deposited, as shown in Fig. 9. Then, clearance holes were processed for the aluminum pads. $30 \mathrm{~nm}$ chromium and $100 \mathrm{~nm}$ gold were evaporated onto the whole area as a plating base. Next, a resist mask was applied and $8 \mu \mathrm{m}$

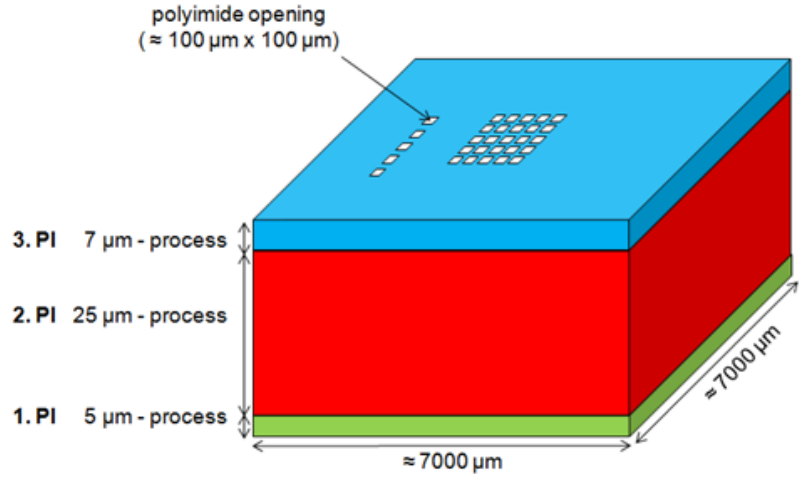

Figure 9: Embedding the dummy stimulator chip into the polyimide.

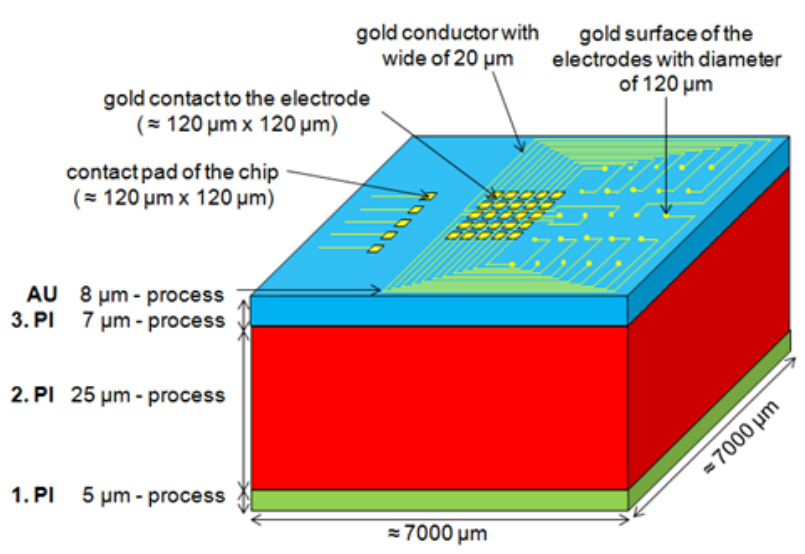

Figure 10: Contacting the dummy stimulator chip to the gold surfaces of the electrodes.

gold was electroplated. The resist mask was removed with acetone, and the plating base was etched wet chemically. The gold pads and the gold surfaces of the electrodes finally remained as shown in Fig. 10.

The gold surfaces were then coated with the electrode material, here iridium oxide $\left(\operatorname{IrO}_{x}\right)$, which is biocompatible and biostable [4]. It is used worldwide by many research groups, since it is adequate for stimulating neurons with a charge-transfer-capacity of up to $95 \mathrm{mC} / \mathrm{cm}^{2}$ after electrochemical activation [5]. Iridium oxide is a highly porous material. To block corrosion of the gold surfaces, platinum (Pt) was used as an intermediate layer. Deposition of $150 \mathrm{~nm}$ platinum and $500 \mathrm{~nm}$ iridium oxide is a proven standard process, and was performed in a sputtering system. For subsequent deposition and patterning of the iridium oxide electrodes, the structures were protected by a negative mask while the excess material was removed using the lift-off method. The resist mask was subsequently removed with hot N-Methyl-2-pyrrolidon. After successful structuring, Flex-MEA looks like in Fig. 11.

Then, parylene C (PA) was used for electrical isolation and for generating well-defined electrode openings of $100 \mu \mathrm{m}$ in diameter (Fig. 12). It was separated 


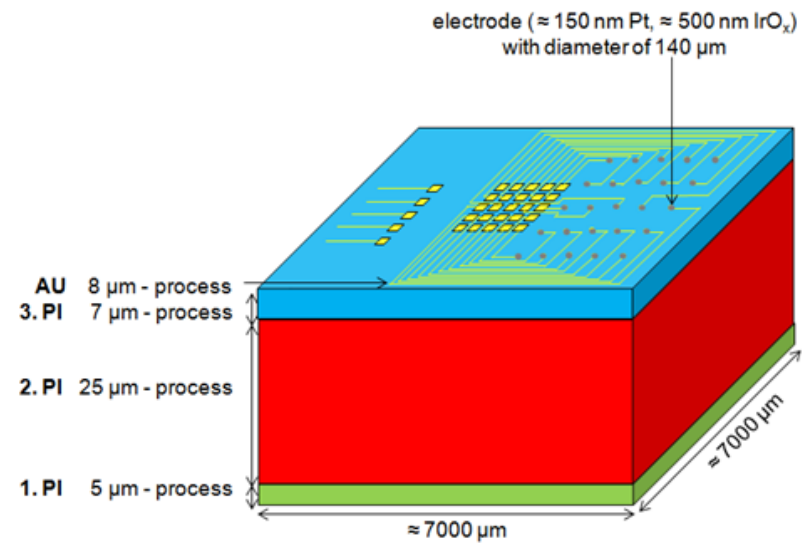

Figure 11: Flex-MEA with iridium oxide electrodes.

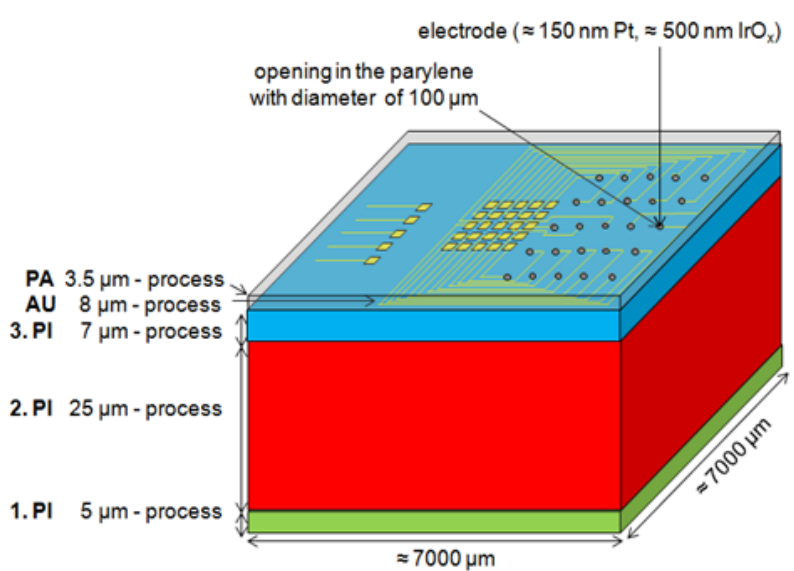

Figure 12: Flex-MEA (schematic).

from the gas phase in a $3.5 \mu \mathrm{m}$-process, and can be dry etched afterwards. Parylene $\mathrm{C}$ is widely used as coating material for medical applications [6].

Next, the structure was separated from the alu-subwafer. First, $150 \mathrm{~nm}$ titanium was etched between the individual structures. Then the entire sacrificial layer was etched, and the Flex-MEAs were detached from the carrier wafer. Finally, the remaining titanium was etched away. During these etch processes, the structures were protected with a resist. A photograph of the finalized Flex-MEA is shown in Fig. 13.

\section{Experiments}

Bending tests were performed to prove the flexibility of the structures. First, Flex-MEA was bent around a pen with a radius of $5 \mathrm{~mm}$ (Fig. 14, left). The microscope pictures show, that the integrated dummy stimulator chip had no visible fracture traces afterwards (Fig. 15, left).

Then, Flex-MEA was bent around a ball pen refill with a small radius of $1.5 \mathrm{~mm}$ (Fig. 14, right). Unfortunately, fracture traces were visible after this demanding stress test (Fig. 15, right).

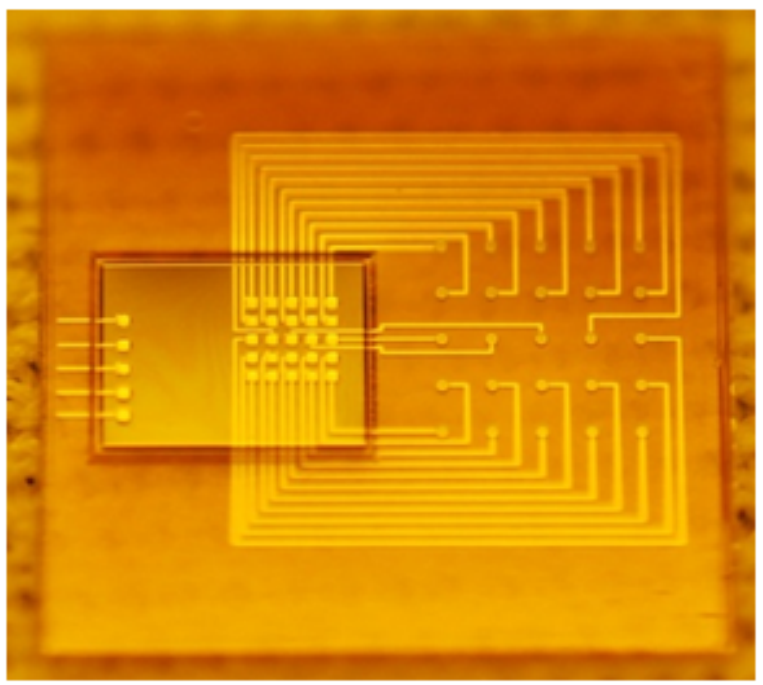

Figure 13: Flex-MEA.

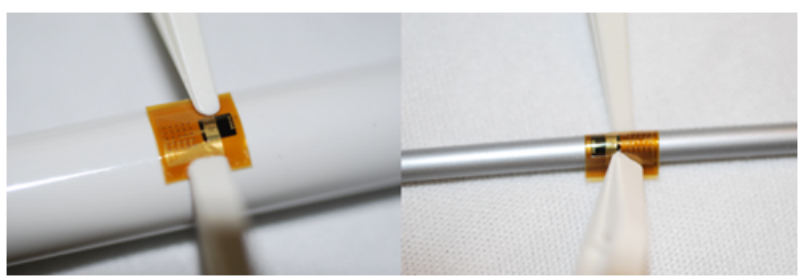

Figure 14: Bending tests with a pen ( $5 \mathrm{~mm}$ radius) and with a ball pen refill $(1.5 \mathrm{~mm}$ radius $)$.
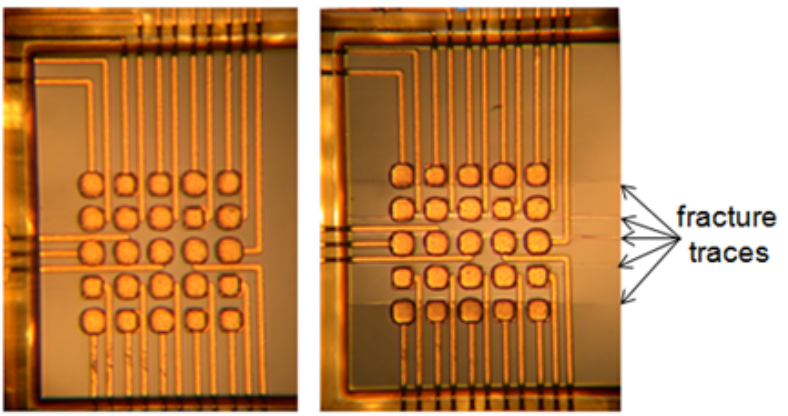

Figure 15: Microscope pictures of Flex-MEA after being bent around the pen (left) and around the ball pen refill (right).

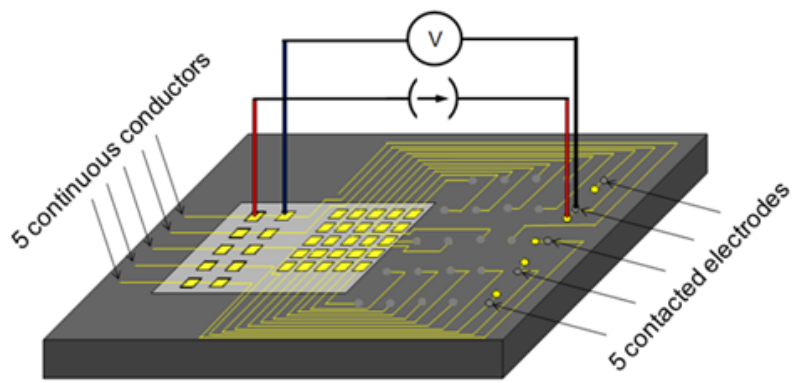

Figure 16: Modified Flex-MEA. 
To prove the robustness of the gold conductors against mechanical stress, a modified version of FlexMEA was designed. The design adds 5 gold conductors connecting the 5 control pads on the left side with 5 electrode pads on the right side, see Fig. 16. Ten additional pads facilitate measurements of the ohmic resistance of these 5 conductors with the fourpoint measurement method, during or after mechanical stress. These measurements are still ongoing.

\section{Conclusion}

A novel flexible multi-electrode array with embedded silicon chips has been manufactured. Mechanical stress tests were carried out, giving promising results. Electrical measurements of the ohmic resistances of the integrated conductors during or after mechanical stress will follow soon.

\section{Acknowledgements}

The research described in this paper was supervised by Prof. W. Mokwa, RWTH Aachen University, and was supported by the German Federal Ministry of Education and Research (BMBF), grant No. 16SV5322K.

\section{References}

[1] E. Margalit, M. Maia, J. D. Weiland et al. Retinal prosthesis for the blind. Survey of Ophthalmology 47(4):335-356, 2002.

[2] W. Mokwa. Retinal implants to restore vision in blind people. In 16th International Conference on Solid-State Sensors, Actuators and Microsystems, TRANSDUCERS 2011, Beijing, 2011, pp. 28252830 .
[3] W. Mokwa, M. Goertz, C. Koch et al. Intraocular epiretinal prosthesis to restore vision in blind humans. In 30th IEEE Annual International Conference: Engineering in Medicine and Biology Society, EMBS 2008, Vancouver, 2008, pp. 5790-5793.

[4] L. S. Robblee, J. L. Lefko, S. B. Brummer. Activated Ir: An electrode suitable for reversible charge injection in saline solution. Journal of the Electrochemical Society 130(3):731-733, 1983.

[5] E. Slavcheva, R., Vitushinsky, W. Mokwa et al. Sputtered iridium oxide films as charge injection material for functional electrostimulation. Journal of the Electrochemical Society 151(7):E226-E237, 2004.

[6] Speciality Coating Systems. SCS medical coatings. http://scscoatings.com/docs/biostabl. pdf, [2011-03-03], 2010.

[7] T. Stieglitz, H. Beutel, M. Schuettler et al. Micromachined, polyimide-based devices for flexible neural interfaces. Biomedical Microdevices 2(4):283-294, 2000.

[8] N. Winkin. Flexible multi-electrode array for medical applications. In 16th International Student Conference on Electrical Engineering, POSTER 2012, Prague, 2012.

[9] J. L. Wyatt, J. F. Rizzo, A. Grumet et al. Development of a silicon retinal implant - Epiretinal stimulation of retinal ganglion-cells in the rabbit. Investigative Ophthalmology $\&$ Visual Science 35(4):1380, 1994. 